\title{
Minimally Invasive Sinus Membrane Elevation Techniques: A Literature Review
}

\author{
Shilpy Bhandari, ${ }^{1}$ Rucha Shah, ${ }^{2 *}$ Raison Thomas, ${ }^{3}$ Tarun Kumar AB, ${ }^{3}$ Mehta DS \\ ${ }^{1}$ Private practitioner, Hyderabad, Telangana, India \\ ${ }^{2}$ Reader, Department of Periodontics, Bapuji Dental College \& Hospital, India \\ ${ }^{3}$ Professor, Department of Periodontics, Bapuji Dental College \& Hospital, India
}

\begin{abstract}
Sinus augmentation surgery has become a well-accepted pre-prosthetic procedure for placement of endo-osseous implants in compromised posterior edentulous maxilla. Various procedures for bone augmentation have been used to achieve sufficient bone volume for dental implants with the lateral and crystal surgical approaches being the most common. But these bone augmentation methods, including the conventional lateral maxillary approach and sinus elevation by osteotome, have several limitations. Sinus membrane perforation is one of the most common complications of the sinus lift procedure. Thus, to overcome these limitations various minimally invasive techniques have been introduced. These techniques cause minimal postoperative pain, faster recovery and are more economical due to shorter remission period. Hence, the aim of this review article is to discuss the various minimally invasive maxillary sinus floor elevation techniques introduced.
\end{abstract}

Keywords: Sinus augmentation, Hydraulic pressure, Gel pressure, Antral balloon elevation, Sinus perforation, Lateral approach, Crestal approach sinus elevation

Abbreviations: BAOSFE, Bone Added Osteotome Mediated Sinus Floor Elevation; MIAMBE, Minimally Invasive Antral Membrane Balloon Elevation; AMBE, Antral Membrane Balloon Elevation; HPMC, Hydroxy Propyl Methyl Cellulose; CE, Cutting Edge; RE, Reamer Edge; CPS, Calcium Phosphosilicate; CADCAM, Computer Aided Design \& Computer-Aided Manufacturing

\section{Introduction}

Edentulous maxillary posterior region has several unique and demanding anatomical features which makes it a challenging area to deal with. ${ }^{1}$ Long standing edentulism in sinus region has several consequences including resorption of available bone and pneumatisation of sinus. Sinus floor elevation is an internal augmentation of maxillary sinus wherein, maxillary sinus membrane (Schneiderian membrane) is elevated to place implants through the sinus floor. ${ }^{2}$

In the last few years, sinus membrane elevation and subsequent implant placement has become an established pre-prosthetic procedure .Various procedures for bone augmentation have been used to achieve sufficient bone volume for dental implants with the lateral and crestal surgical approaches being the most common (Figure
1). ${ }^{3}$ Tatum was the first person to introduce lateral approach in the late 1970s and first published report in literature was by Boyne \& James in 1980.,5 Later in 1994, summer described a novel transalveolar technique using a set of osteotomes with varying diameters. ${ }^{6}$ This technique was further modified by including the graft material into the osteotomy and is known as bone-added osteotome sinus floor elevation (BAOSFE) or "Summers technique"(Figure 1). It was considered to be less traumatic and more conservative than the lateral approach. ${ }^{7}$

However, both the lateral and crestal approaches for sinus elevation have several shortcomings. The most commonly experienced complication is sinus membrane perforation. Increased morbidity \& postoperative pain, increased chair side time, high cost of

\begin{tabular}{|l|l|}
\hline Quick Response Code: & *Corresponding author: Rucha Shah, Department of Periodontics, Bapuji Dental College \& \\
Hospital, MCC B Block, Davangere, Karnataka, India \\
Received: 02 March, 2021 Published: 26 March, 2021 \\
Citation: Shilpy B, Rucha S, Raison T, Tarun KAB, Mehta DS. Minimally Invasive Sinus Mem- \\
brane Elevation Techniques: A Literature Review. SOJ Den Oral Disor. 2021;1(2):1-6. D0I: \\
10.53902/SOJDOD.2021.01.000507
\end{tabular}


the procedure \& vertigo in few cases are various other limitations that these techniques present. To overcome these limitations and to make the procedure of sinus membrane elevation minimally invasive various indigenous techniques have been introduced. ${ }^{8}$

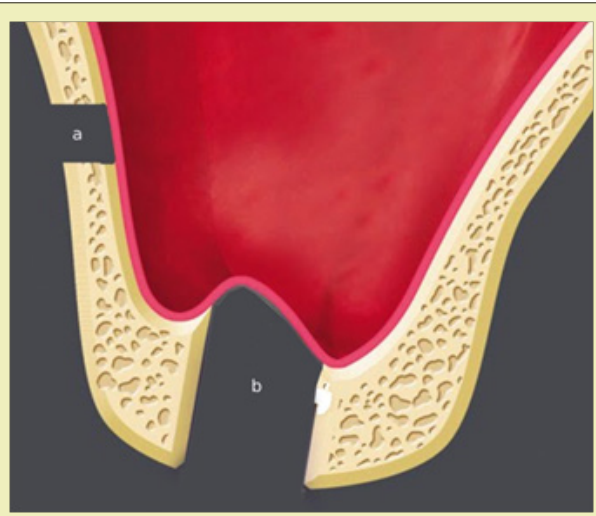

Figure 1: (A) Conventional crystal approachsinuselevationtechnique. (B) Conventional lateral approach sinus elevation technique.

Minimally invasive surgical techniques are procedures with minimal damage and trauma to biological tissues. ${ }^{9}$ These techniques cause minimal postoperative pain, morbidity, faster recovery and are more economical due to shorter remission period. Several such techniques have been proposed in the last few years as safer \& effective alternative to the conventional sinus floor elevation techniques. The aim of this article is to discuss the various minimally invasive techniques for maxillary sinus floor elevation.

\section{Minimally Invasive Techniques for Maxillary Sinus Floor Elevation}

Minimally invasive membrane elevation has been tried by various methods such as using balloon elevation, hydraulic pressure, gel pressure, negative pressure, piezoelectric system, reamer mediators, using CPS putty, and CAD- CAM. The details of the following are mentioned below. ${ }^{10}$

\section{a) Minimally invasive antral membrane balloon el- evation technique (MIAMBE)}

It was first introduced by Kfir et al as a modification of antral membrane balloon elevation (AMBE) to overcome various limitations like buccal window preparation and larger incisions (Figure 2). ${ }^{11,12}$ In this technique, mucoperiosteal flap is elevated at the edentulous space under local anaesthesia. Osteotomy on the crestal bone is prepared using $2 \mathrm{~mm}$ diameter drill up to the depth as determined in the computed tomography scans. Drilling is stopped $1-2 \mathrm{~mm}$ below the sinus floor. Using osteotomes, $2 \mathrm{~mm}$ osteotomy site is expanded and bone graft material is inserted. Consequently, sinus floor is in fractured using osteotomes and screw taps. Following this, sinus membrane integrity is evaluated and metal sleeve of the balloon harbouring device was introduced into the osteotomy site. Using barometric inflator, balloon is inflated up to two atmospheric pressures. Upon expansion of balloon beneath the sinus membrane, pressure drops down to 0.5 atmospheres. Gradual inflation of balloon with contrast fluid elevates the sinus membrane.
In order to prevent the withdrawal of elevated sinus membrane, inflated balloon is kept inside the oral cavity for 35 minutes even after attaining the desired sinus elevation. This is followed by deflation \& removal of balloon and placement of graft in the space created. (Figure 2) Simultaneous implant placement can be done. Authors suggest that this technique is safe \& effective means of sinus elevation. It is easy to perform \& requires short learning curve. ${ }^{11}$ However, during balloon inflation the main force is directed upward and peak stresses may act on the membrane which may sometimes cause membrane perforation, serving as a drawback of this technique.

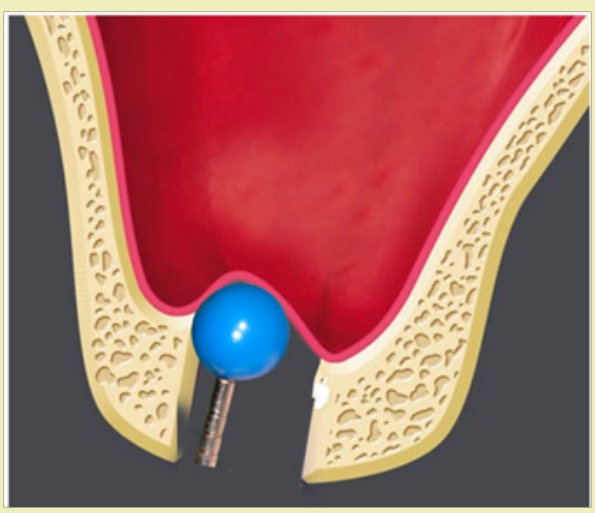

Figure 2: Minimally invasive antral membrane balloon elevation.

\section{b) Hydraulic pressure to elevate sinus membrane}

Chen \& Cha in the year 2005 published a study on sinus elevation with simultaneous implant placement utilizing sinus condensing kit. ${ }^{13}$ In this procedure, a crestal incision is given under local anaesthesia in the edentulous area. Selection of the round bur is done based on the width of the implant to be placed. Drilling is performed leaving $1 \mathrm{~mm}$ from of sinus floor. This is followed by selection of narrow sized burs in order to create a conical shape osteotomy end. A $2 \mathrm{~mm}$ of round bur is used to create a pinhole on the sinus floor \& hydraulic pressure is delivered through the high speed hand piece which aids in sinus membrane separation (Figure 3). Further elevation of the membrane takes place with the help of bone graft mixture which is pushed through the pinhole by a sinus condenser. Once the sufficient amount of graft is introduced to provide a cushioning effect \& minimize the membrane tear, implant is drilled followed by primary closure.

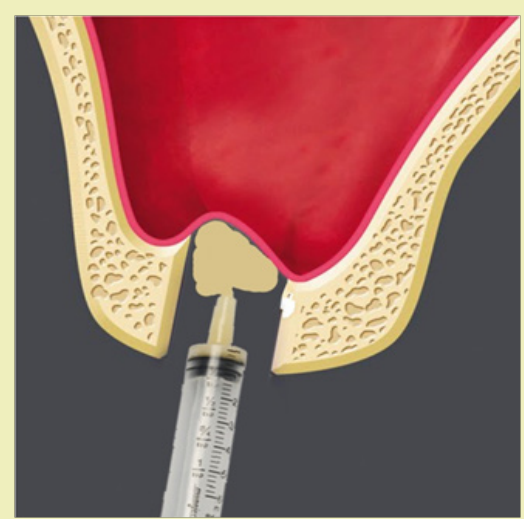

Figure 3: Minimally invasives in us membrane elevation using hydraulic pressure. 
Another study by Sotirakis \& Gonshor in the same year 2005, described a similar technique using hydraulic pressure to elevate the sinus membrane. It was first modelled experimentally in hen eggs, and then in human cadaver preparations. According to authors this technique was amalgamation of advantages of lateral approach which allowed high volume of graft material along with ease of crestal approach technique. In this technique, a syringe filled with saline was adjusted to the osteotomy site and an airtight interface is maintained between bone \& syringe. The membrane was detached and elevated with the help of hydraulic pressure created by depression of the plunger of the syringe. The authors suggest that their technique is simple and quick. Less laborious as larger flap reflection \& buccal window preparation are avoided and also has an additional advantage of minimal postoperative patient morbidity. ${ }^{10}$

\section{i. Water lift system}

Kim, Itoh \& Kang in the year 2012 published a novel technique using water lift system for sinus floor elevation. ${ }^{2}$ This rationale for the use of this system is based on Pascal principal according to which pressure applied to an enclosed fluid is transmitted equally to every part of the fluid. For the direct sinus lift procedure, lateral approach kit is utilized. ${ }^{2}$

In this, crestal approach water lift system is utilized. The osteotomy site is created using a conventional drill. Subsequently, the compaction drill is used to compact the remaining bone. The osteotomy site is drilled using artificially intelligent (AI) drill and using aqua injector, radiographic contrast medium was injected through the osteotomy site to elevate the sinus membrane. The amount of elevation achieved during the procedure is assessed using X ray. After adequate elevation of membrane, the osteotomy site is enlarged, the sinus is grafted and successively implant is placed. ${ }^{2}$

\section{ii. Sinus Membrane elevation by gel pressure}

This technique was introduced by Pommer \& Watsek in 2009 in a cadaver study. ${ }^{14,15} \mathrm{~A}$ surgical template is prepared \& placed on the ridge. Mucoperiosteal flap is not reflected instead a soft tissue punch of $4.1 \mathrm{~mm}$ diameter is used at intended site on the crest. Following this, bony floor is fractured using cannon drills of $3.3 \mathrm{~mm}$ diameter with internal irrigation. Drilling depths of cannon drills are regulated using custom made drill stops. Further, a specially designed injection nozzle with a radiopaque gel was introduced into the osteotomy site to elevate the sinus membrane (Figure 3). The gel consists of $2 \%$ Hydroxy propyl methyl cellulose (HPMC), a viscoelastic agent, $37 \%$ iopamidol, a radio opaque marker mixed at a ratio of 3:1.

\section{c) Transcrestal approach with CPS putty}

This technique was introduced by Kher \& co workers in 2014 who utilized calcium phosphosilicate (CPS) putty for hydraulic sinus membrane elevation. In this technique after the reflection of full thickness mucoperiosteal flap following crestal incision in edentulous space in maxilla, an osteotomy site is created using $2 \mathrm{~mm}$ drill. Drill is stopped $1 \mathrm{~mm}$ short of the sinus floor as estimated with the help of radiograph. Further widening of the osteotomy site is done using different drills. Around $0.2 \mathrm{~cm}$ of Calcium silicophosphate putty is supplied into the osteotomy using a catridge. This acts as cushion \& subsequently osteotomes \& mallets with depth markings are introduced to produce greenstick fracture of the sinus floor. Following this bone substitute i.e $0.5 \mathrm{~cm}$ CPS are inserted into the cavity through catridge delivery system. Which induces a hydrostatic pressure and helps in the elevation of the sinus membrane? Once the elevation of membrane is achieved, implant is placed. Authors suggest that this technique possess minimal risk of perforation due to consistency of putty. Timely replacement of CPS allows replacement with new bone. It shows better implant stability due to rapid turnover rate upon usage of CPS. Further, this technique can be utilized in areas with minimal residual ridge height and have shown considerable gain in bone height comparable to lateral approach. However an experienced operator is needed to perform this technique sensitive procedure. It also requires minimum of $3 \mathrm{~mm}$ of residual bone for sinus lift with simultaneous implant placement in order to achieve primary stability in implants placed. ${ }^{16}$

\section{d) Piezoelectric Minimally Invasive System}

Piezoelectric instruments work in a way that only enables them to cut hard tissue without causing ant damage to adjacent soft tissues (Figure 4). This technique has been introduced by Vercelloti et al. ${ }^{17}$ Troedhan et al in conjunction with aceton group have developed the intralift technique. ${ }^{18}$ In this technique, crestal osteotomy is performed according to the sequence of the Intralift ${ }^{\mathrm{TM}}$ system. The preparation of the hole to access the floor of the sinus floor is performed at the crest. Four power modes are available D-1 to D-4, which correspond to bone quality. The D-1, D-2 power are used first and correspond to cortical bone, whereas D-3, D-4 correspond to cancellous bone and are used later. The separation of the periosteum is achieved by the help of ultrasonic vibrations and also by hydro-pneumatic pressure of saline solution as created by the piezoelectric cavitation. ${ }^{18}$

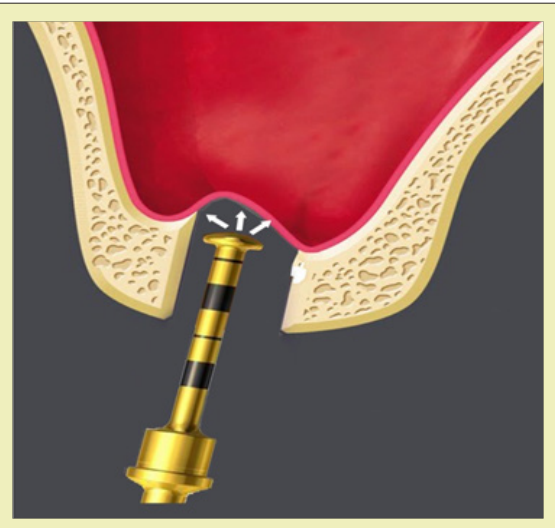

Figure 4: Minimally invasive sinus membrane elevation sing gel pressure.

\section{e) Reamer mediated sinus floor elevation}

This was introduced by Ahn \& co workers wherein they used a specially designed reamer with one cutting edge (CE) at 85 degree cutting angle to elevate the sinus membrane ${ }^{19}$. In this technique, after reflection of mucoperiosteal flap following crestal incision in 
the edentulous space, the implant site is marked using $2 \mathrm{~mm}$ round bur. Initially a $2 \mathrm{~mm}$ reamer is used to drill the osteotomy site to a depth of $1 \mathrm{~mm}$ followed by series of reamers to enlarge the site. Depending on the bone density, diameter of final reamer used is 0.5 $1.5 \mathrm{~mm}$ less than the implant diameter. With the help of hand piece the contact between the reamer and the sinus floor is established at 50rpm. Once the bony resistance is lost after reaming, end probe is used to release the membrane from the underlying sinus floor and patency of the membrane is checked. With the help of a blunt end condenser graft material is added and displaced apically $2 \mathrm{~mm}$ deeper than residual bone height with final reamer at $30 \mathrm{rpm}$. Successive condensation of graft material is done until desired height for implant placement is attained. ${ }^{19}$

The authors suggest that the basic action mechanism of the reamer comes from its one edged blade situated at a specific angle (85degree). The CE performs the primary bone cutting and makes the bony hole circular. The cutting angle creates the angle between the cutting edge (CE) and the tip of the reamer and provides the cutting function. The reamer edge (RE) removes the remaining bone in the osteotomy laterally 180degrees behind the CE. In addition, the flat end of the RE performs a light vertical pushing action on the sinus floor during the reaming and the groove removes the bone chips. These actions make a round-form bone shell on the cortical bone of the sinus floor. ${ }^{19}$

\section{f) CAD CAM}

Pozzi \& Co- workers 2013.described a procedure for sinus elevation using computer guided planning and guided surgical approach through the use of computer aided design (CAD)/computer-aided manufacturing (CAM)-generated surgical template in combination with expander-condensing osteotomes. ${ }^{20}$ In this technique, stereo lithographic assisted surgical template is fabricated as per the planned implant locations. Drills and calibrated expanding condensing osteotomes are used through the surgical template. The sinus floor is in fractured using condensing osteotomes. Sinus membrane elevation is achieved by the virtue of hydraulic pressure exerted by the graft material and also by compression of the osteotomes. $^{20}$

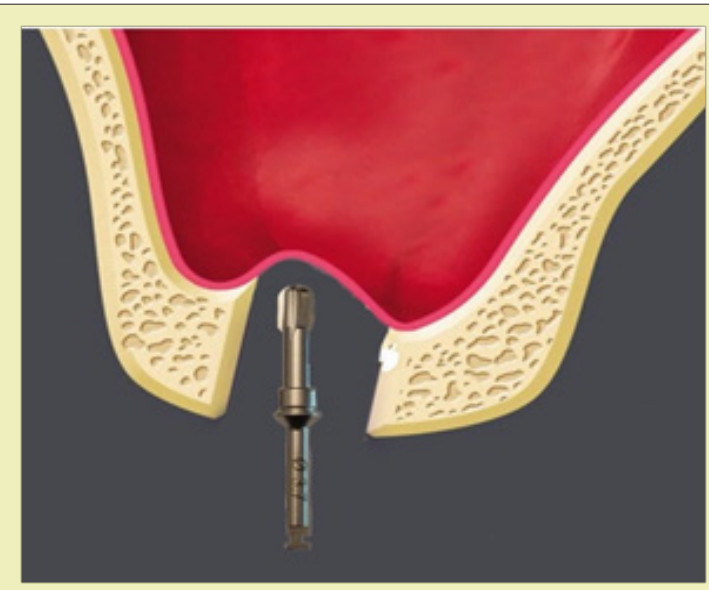

Figure 5: Minimally invasive sinus membrane elevationus in greamer.

\section{Discussion}

Long term edentulism in the posterior maxilla leads to resorption of bone and pneumatisation of sinus. Amalgamation of both along with poor quality bone turns out to be a major challenge for implant placement in the region. ${ }^{21}$ In the era of prosthetically driven implant dentistry, such situations are no more an obstacle in the implant placement. Sinus lift procedure includes elevation of sinus membrane to a certain height which allows the placement of implants through the sinus floor without damaging the integrity of sinus membrane. Tatum in late 1970s \& summers in 1991 were the pioneers of direct \& indirect sinus lift procedures respectively. These procedures were described in 1970's and since then, lot of advancements have been proposed to carry out sinus lift procedure. These advancements proposed aim to make procedures more accurate, less technique sensitive, decrease patient morbidity \& chair side time. ${ }^{12}$ In a longitudinal study of 359 sinus lift procedures by Nolan \& co workers for 3 years,

7 out of every 10 failed sinus grafts were accompanied by a perforated Schneiderian membrane during sinus lift surgery. ${ }^{22}$ According to authors, the risk of incidence of sinusitis increased with membrane perforation. After sinus membrane perforations were investigated by several authors. It was observed that there is decrease in implant survival rates after the incidence of membrane perforation. ${ }^{23,24}$ However, few other studies did not report any significant differences in implant survival rates between the perforated and non-perforated side..$^{25-30}$

Thus, to overcome the challenges of conventional sinus lift procedures and minimize the risk of membrane perforations, these various minimally invasive techniques were introduced. Any surgical technique which causes minimal damage to the biological tissues is known as minimally invasive procedure. These procedures comprise of techniques with smaller incisions which not only reduce the risk of membrane perforation but also minimize the trauma to the tissues and subsequently reducing postoperative patient morbidity. ${ }^{31}$ These techniques possess various advantages such as minimal postoperative pain, faster recovery $\&$ are more economical and higher patient satisfaction.

Several minimal invasive techniques have been developed to improve the results of conventional sinus lift procedures. Kfir et al. ${ }^{11}$ worked on the minimally invasive antral membrane balloon elevation (MIAMBE) technique wherein integrity of sinus membrane is maintained by gradual elevation with the help of a balloon. In a multicenter research study in 109 patients, 95\% of implant survival rate with only 3 cases with sinus membrane perforation were reported. It was concluded that this technique can be employed as alternative to conventional techniques. ${ }^{11}$ Chen \& $\mathrm{Cha}^{13}$ reported a technique using hydraulic pressure for minimally invasive sinus floor elevation. According to authors this technique is advantageous as it provide greater tactile control, a traumatic as cortical bone is perforated than fractured. Also, in cases of sloping sinuses \& compartmental sinus septum this technique is beneficial as it makes use of a pinhole through which only hydraulic pressure 
and graft material is pushed. ${ }^{13}$ However one of the drawback encountered is the risk of membrane perforation as the use of a fluid jet may cause pressure peaks at sites where the jet impacts the Schneiderian membrane. ${ }^{2}$ Kim \& co- workers introduced water lift system which was found to be effective. This system is effective in minimizing rates of sinus membrane perforations due to usage of a specialized AI drill which is sensitive to bony resistance. There by decreasing chances of membrane perforations. Also, the rate of delivery of water uniformly distributes hydraulic pressure. ${ }^{2}$ There are no point transmission of forces/ stresses as seen in osteotome mediated sinus elevation leading to minimal risk of perforation. ${ }^{14}$ Along with this, technique is less technique sensitive and requires less chair side time with greater precision. However, the drawbacks of this system include high cost of the kit \& cautions during usage of AI drill in crestal approach. ${ }^{2}$

Pommer and Watzek executed transcrestal sinus lift procedure in 10 atrophic maxillae of human cadavers. The advantages of this technique are that usage of gel allow smooth and transmit forces in greater areas. Also, the gel provides cushioning effect to the sinus membrane by absorbing sudden pressure, thereby minimizing the risk of membrane perforation. Moreover, flapless approach provides greater vascularisation to the graft \& minimizes bone resoprtion. However, limited evidence available \& more studies are required to assess the technique. ${ }^{15}$ Thus authors claimed this technique as an substitute to conventional techniques15. Another study by Suguimoto \& co-workers a negative pressure is created inside the sinus cavity with the help of a suction tube which is positioned over the opening created superiorly \& distally to the lateral window. This causes separation of the sinus mucosa from the underlying bone and further towards the lateral wall of the sinus. ${ }^{32}$ But the main drawback is that it is technically more sensitive and requires an experienced operator.

Another minimally invasive technique makes use of piezoelectric ultrasound technology which is developed to simplify the conventional technique \& minimize trauma to the patients. However, excessive mechanical forces may cause perforation of membrane and may also cause excess heat generation during the long surgical procedure. Ahn and co workers performed minimally invasive reamer mediated transalveolar sinus floor elevation technique. Authors suggested that it is a safe \& effective technique. It has more availability of autogenous bone due to absence of irrigation system. However this technique is less tactile sensitive. ${ }^{19}$

Pozzi \& co-workers performed CAD CAM technique. The advantages to the transcrestal guided sinus lift technique include negligible bone resorption as there is no flap elevation. Also, allows greater vascularise to the alveolar ridge \& graft material. It is easy to execute, a traumatic, low postoperative patient morbidity with enhanced patient acceptance for the procedure seen. However this technique is highly expensive. Also thorough knowledge \& familiarity with the technology are major requisites for an operator to perform this technique. Together these techniques have consistently shown a reduction in the rate of sinus membrane perforations \& re- duction in the stress associated with the traumatic, time consuming \& laborious conventional procedures. These techniques not only reduce the chair side time but also are less technique sensitive, have short learning curves, are safe \& easy to execute. However, these techniques are associated with some drawbacks like high costs of the kits, requirement of skilled operators with greater precision to perform some of the procedures which are technique sensitive. Future studies should focus on development of techniques which would be more effective in overcoming the limitations of the current techniques.

\section{Conclusion}

In the era of patient friendly and minimally invasive dentistry, The minimally invasive sinus floor elevation techniques provide an exciting alternative to conventional sinus elevation techniques. Based on the benefits and indications for usage in the patients, operator can make use of these techniques to the best of his abilities to derive the successful results. However, there is limited literature available on these techniques. Hence more longitudinal \& long term multicenter studies are required to further substantiate their effectiveness.

\section{Acknowledgments}

None.

\section{Funding}

None.

\section{Conflicts of interest}

Author declares that there is no conflict of interest.

\section{References}

1. Corbella S, Taschieri S, Del Fabbro M. Long term outcomes for the treatment of atrophic posterior maxilla: a systematic review of literature. Clin Implant Dent Relat Res. 2015;17(1):120-132.

2. Kim DY, Itoh Y, Kang TH. Evaluation of the Effectiveness of a water lift system in the sinus membrane-lifting operation as a sinus surgical instrument. Clin Implant Dent Relat Res. 2012;14(4):337-347.

3. Petruzzi M, Ceccarelli R, Testori T, et al. Sinus floor augmentation with a hydro pneumatic technique: a retrospective study in 40 patients. Int J Periodontics Restorative Dent. 2012;32(2): 205-210.

4. 4.Tatum H Jr. Maxillary and sinus reconstructions. Dent Clin North Am. 1986;30(2):207-229.

5. Boyne PJ, James R.A. Grafting of the maxillary sinus floor with autogenous marrow and bone. J Oral Surg. 1980;38(8):613-616.

6. Summers RB. A new concept in maxillary implant surgery: the osteotome technique. Compendium. 1994 ;15(2):152,154-156.

7. Summers RB. Sinus floor elevation with osteotomes. J Esthet Dent. 1998;10(3):164-171.

8. Vernamonte S, Mauro V, Messina AM. An unusual complication of osteotome sinus floor elevation: benign paroxysmal positional vertigo. Int J Oral Maxillofac Surg. 2011;40(2):216-218.

9. Wickham JE. The new surgery. $B r$ Med $J$ (Clin Res Ed). 1987;295(6613):1581-1582.

10. Sotirakis EG, Gonshor A. Elevation of the maxillary sinus floor with hydraulic pressure. J Oral Implantol. 2005;31(4):197-204. 
11. Kfir E, Goldstein M, Yerushalmi I, et al. Minimally invasive antral membrane balloon elevation - results of a multicenter registry. Clin Implant Dent Relat Res. 2009;11 Suppl 1:e83-91.

12. Soltan M, Smiler DG. Antral membrane balloon elevation. JOral Implantol. 2005;31(2):85-90.

13. Chen L, Cha J. An 8-year retrospective study: 1,100 patients receiving 1,557 implants using the minimally invasive hydraulic sinus condensing technique. J Periodontol. 2005;76(3):482-491.

14. Pommer B,Unger E. Biomechanics of transcrestal sinus membrane elevation. In: George Watsek, ed. The Percrestal Sinuslift-From Illusion to Reality. $1^{\text {st }}$ ed. Quintessence: 2012:87-95.

15. Pommer B, Watzek G. Gel-pressure technique for flapless transcrestal maxillary sinus floor elevation: a preliminary cadaveric study of a new surgical technique. Int J Oral Maxillofac Implants. 2009;24:817-822.

16. Kher U, Ioannou AL, Kumar T Siormpas K, et al. A clinical and radiographic case series of implants placed with the simplified minimally invasive antral membrane elevation technique in the posterior maxilla. J Craniomaxillofac Surg. 2014;42(8):1942-1947.

17. Vercellotti T, De Paoli S, Nevins M. The piezoelectric bony window osteotomy and sinus membrane elevation: introduction of a new technique for simplification of the sinus augmentation procedure. Int J Periodontics Restorative Dent. 2001;21(6):561-567.

18. Troedhan AC, Kurrek A, Wainwright M, et al. Hydrodynamic ultrasonic sinus floor elevation--an experimental study in sheep. J Oral Maxillofac Surg. 2010;68(5):1125-1130.

19. Ahn SH, Park EJ, Kim ES. Reamer-mediated transalveolar sinus floor elevation without osteotome and simultaneous implant placement in the maxillary molar area: clinical outcomes of 391 implants in 380 patients. Clin Oral Implants Res. 2012;23:866-872.

20. Pozzi A, Moy PK. Minimally invasive transcrestal guided sinus lift (TGSL): a clinical prospective proof-of-concept cohort study up to 52 months. Clin Implant Dent Relat Res. 2014;16(4):582-593.

21. Woo I , Le BT. Maxillary sinus floor elevation: review of anatomy and two techniques. Implant Dent. 2004;13(1):28-32.
22. Nolan PJ, Freeman K, Kraut RA. Correlation between Schneiderian membrane perforation and sinus lift graft outcome: a retrospective evaluation of 359 augmented sinus. J Oral Maxillofac Surg. 2014;72(1):47-52.

23. Hernandez-Alfaro F, Torradeflot MM, Marti C. Prevalence and management of Schneiderian membrane perforations during sinus-lift procedures. Clin Oral Implants Res. 2008;19(1):91-98.

24. Proussaefs P, Lozada J, Kim J, et al. Repair of the perforated sinus membrane with a resorbable collagen membrane: a human study. Int J Oral Maxillofac Implants. 2004;19(3):413-420.

25. Cha HS, Kim A, Nowzari H, et al. Simultaneous sinus lift and implant installation: prospective study of consecutive two hundred seventeen sinus lift and four hundred sixty- two implants. Clin Implant Dent Relat Res. 2014;16(3):337-347.

26. Ding X, Zhu XH, Wang HM, et al . Effect of sinus membrane perforation on the survival of implants placed in combination with osteotome sinus floor elevation. J Craniofac Surg. 2013;24(2):e102-104.

27. Froum SJ, Khouly I, Favero G, et al. Effect of maxillary sinus membrane perforation on vital bone formation and implant survival: a retrospective study. J Periodontol. 2013;84(8):1094-1099.

28. Rickert D, Vissink A, Slater JJ, et al. Comparison between conventional and piezoelectric surgical tools for maxillary sinus floor elevation. A randomized controlled clinical trial. Clin Implant Dent Relat Res. 2013;15(2):297-302.

29. Becker ST, Terheyden H, Steinriede A, et al. Prospective observation of 41 perforations of the Schneiderian membrane during sinus floor elevation. Clin oral implants Res. 2008;19(12):1285-1289.

30. Karabuda C, Arısan V, Hakan O. Effects of sinus membrane perforations on the success of dental implants placed in the augmented sinus. $J$ Periodontol. 2006;77(12):1991-1997.

31. Johansson LA. On minimally invasive approaches to sinus lift procedures. 2012.

32. Suguimoto RM, Trindade IK, Carvalho RM. The use of negative pressure for the sinus lift procedure: a technical note. Int J Oral Maxillofac Implants. 2006;21(3):455-458. 